\title{
Enterprise Crowdsourcing Solution for Software Development in an Outsourcing Organization
}

\author{
Ranganathan Jayakanthan and Deepak Sundararajan \\ TCS Innovation Labs - Web 2.0, Tata Consultancy Services, Chennai, India \\ jayakanthan@acm.org, deep.sun@tcs.com
}

\begin{abstract}
Enterprise Crowdsourcing has the potential to be a very powerful and disruptive paradigm for human resource deployment, project development and project management as we know them. This paper details ongoing work at TCS Innovation Labs - Web 2.0, Tata Consultancy Services, Chennai, India to develop an Enterprise Crowdsourcing Solution to tackle the various processes involved in the development of software by leveraging the untapped human resource in the organization. Large IT organizations have a lot of untapped manpower in the form of trainees, the bench strength and people involved in roles which do not fully employ their strengths in particular technologies they are experts in. This system aims to allow untapped talent to get access to challenging tasks part of other projects and work on them while providing a disruptive way to allocate resources in a conventional software development environment.
\end{abstract}

Keywords: Enterprise crowdsourcing, Crowdsourcing, Web 2.0, Collaborative work, Social networking.

\section{Introduction}

Jeff Howe's influential 2006 essay "The Rise of Crowdsourcing" starts off as, "Remember outsourcing? Sending jobs to India and China is so 2003. The new pool of cheap labor: everyday people using their spare cycles to create content, solve problems, even do corporate R \& D." [1] This being 2011, outsourcing and crowdsourcing have both had tremendous growth in the meanwhile. This paper will look into an ongoing project to develop an enterprise crowdsourcing enabler for software development, inside a large outsourcing organization, attempting to fuse Howe's idea posited as an alternative to crowdsourcing, by subsuming it within the larger outsourcing paradigm. We have come full circle, in some sense!

\subsection{Crowdsourcing}

Howe presented developments in the 2006 essay [1] about how distributed, talented amateurs were disrupting the business models of established professionals through mechanisms which allowed them to reach out to potential employers and large business buyers of their products and services, allowing them to provide previously inconceivable amounts of savings in terms of money as well as other resources. Since then, crowdsourcing has grown by leaps and bounds, transforming chimera like into various 
avatars which result in radically different ways of looking at developing solutions to problems with enormous amounts of savings of resources with remarkable efficiencies achieved in attracting the right talent to work on the right problems.

\subsection{Enterprise Crowdsourcing}

Enterprise crowdsourcing posits the use of crowdsourcing in the enterprise to "access scalable workforce on-line." [2] Various organizations have succeeded at enterprise crowdsourcing by adopting various approaches. Organizations as diverse as GoldCorp and Proctor \& Gamble have utilized crowdsourcing to attract the attention of external talent to tackle their hard problems. Enterprise crowdsourcing to attract talent from within the organization has mostly been restricted to uses such as knowledge sharing and idea generation.

In this paper we present an attempt inside a large outsourcing organization with 200,000 employees (and growing) to leverage untapped talent and target them towards software development subtasks. In our organization, as with many other such large outsourcing organizations, a significant percentage of the available human resources remain latent and untapped due to various reasons such as people being unallocated (on bench), in training and in projects in which all their relevant skills are not being utilized. Allocation of people into a project is a rigid, structured process, which involves multiple interviews and evaluation, processes that make it difficult to dynamically allocate resources to tackle smaller projects or problems. We detail a work-in-progress solution to apply enterprise crowdsourcing to outsourced software development.

\section{Crowdsourcing Application}

The TCS crowdsourcing application provides an integrated solution comprising of a marketplace for jobs (tasks), a workflow for creation of jobs, roles for users such as reviewer, requester and freelancer, a reputation mechanism, a virtual currency and a reviewing system. Let us look at some of the system.

\section{$2.1 \quad$ Scope}

The scope of the system is defined to address two types of requirements. The first is to cater to tasks that are generic and internal to the outsourcing company, TCS in this case. The second would be to crowdsource customer specific tasks. While the first is straightforward, the second would require an agreement with the customer and the tasks be made available only to the employees involved in the customer project.

\subsection{Participants and User Roles}

The participants of the system are the employees of TCS. They can be in internal projects, customer projects, undergoing training or in bench waiting for their next assignment.

The users in the system have roles such as requester (where they create jobs, invite participation, and manage jobs), freelancer (where they participate in a particular job) and reviewer (where they review work completed as part of a job.) Reviewers are 
nominated by the job requesters primarily on the basis of their reputation in the category the job was posted in. Users can have multiple roles.

\subsection{Job Creation and Allocation}

Tasks in the system are referred to as jobs. Task might involve a variety of subtasks which are part of the software development process in an outsourced IT firm. A wizard is provided for creating a job in the system. (Fig 1.) The job can be selected from among templates in the system with the ability to represent details dates, milestones and checklists, completion checklist, category and skillset, completion criteria along with the ability to nominate people to the job.

Once the job is created, it will be available in the Job Marketplace for the users to view and apply for the job.

The system will support three modes of job allocation according to the need of the requester. First, the requester will have the ability to nominate a freelancer or pick and choose a freelancer based on the applications received (applies for critical jobs). When there is need for more people to perform similar tasks - testing for instance, the requester can choose to allocate the job in a first come first served basis. Lastly, when the requester wants to get the best solution for a given problem, he can create a competition where any number of freelancers can take up the job and submit their solution.

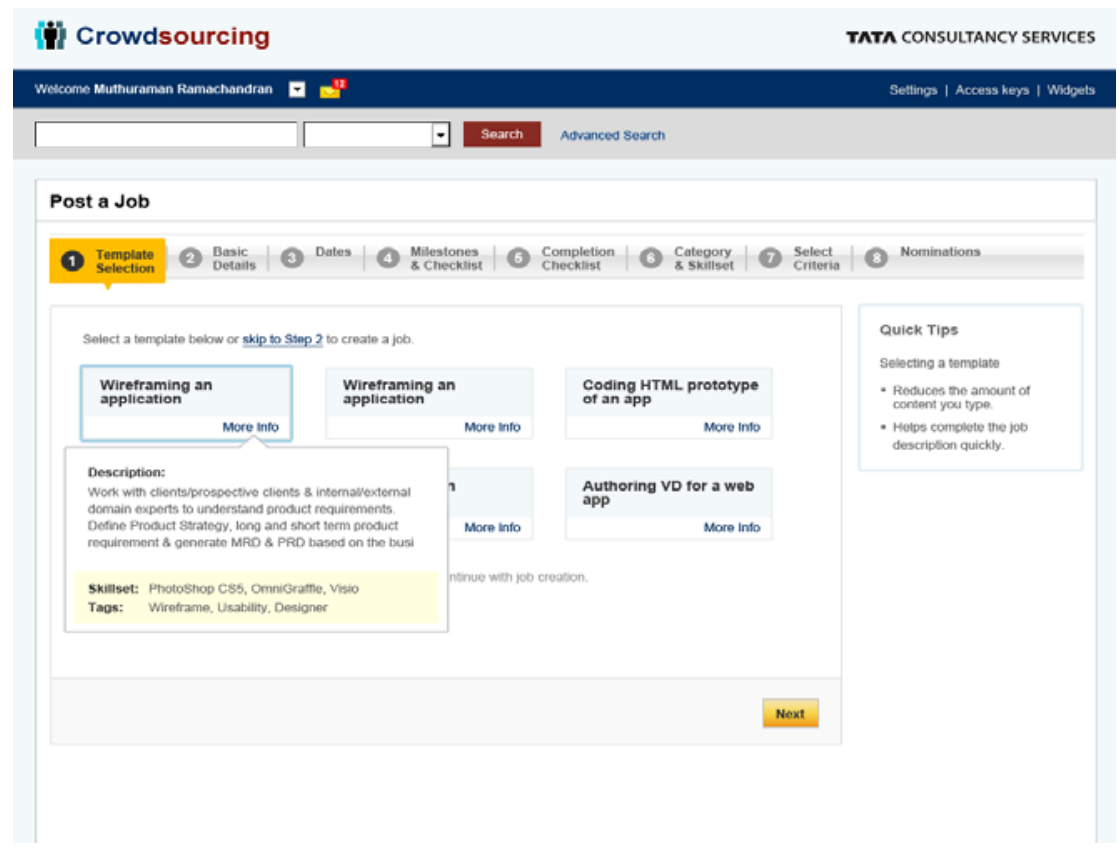

Fig. 1. Job creation wizard 


\section{Conclusion}

The system provides a means to implement a crowdsourcing paradigm to traditional project management through a simplified job creation wizard, a marketplace and a reputation mechanism which apply salient social networking features to this problem. We look forward to deploying this application as a pilot with a selected number of projects in a month.

\section{References}

1. Howe, J.: The Rise of Crowdsourcing. Wired 14(6) (2006)

2. Vukovic, M.: Crowdsourcing for Enterprises. In: 2009 Congress on Services I, pp. 686-692 (2009) 